\title{
ANÁLISE DO PERFIL DE DOCENTES E DISCENTES DA ÁREA DA SAÚDE DA UNIVERSIDADE DO ESTADO DO AMAZONAS QUANTO AO USO DE RECURSOS TECNOLÓGICOS PARA O APRENDIZADO.
}

\author{
MANAUS/AM AGOSTO/2018
}

\author{
Waldeyde Oderilda Magalhães dos Santos - UEA - waldeyde@uol.com.br \\ Marcia S. Lima - UEA - marcia.sampaio.lima@gmail.com \\ Desirée Emelly Dantas Gomes - UEA - desiree.gomes@unasusamazonia.com.br \\ Mayana F. Veras - UEA - mayana.veras@unasusamazonia.com.br \\ Lucas Pessoa Pereira - UEA - lucas.pereira@unasusamazonia.com.br \\ Cael Fernando S. Pinheiro - UEA - cael.pinheiro@unasusamazonia.com.br \\ Alcy Ferreira Magalhães Neto - UEA - afmn.med@uea.edu.br
}

Tipo: Investigação Científica (IC)

Natureza: Descrição de Projeto em Andamento

Categoria: Pesquisa e Avaliação

Setor Educacional: EDUCAÇÃO SUPERIOR, EDUCAÇÃO CONTINUADA EM GERAL

\section{RESUMO}

Este artigo apresenta uma pesquisa exploratória que teve como propósito investigar o perfil dos usuários (docentes e discentes) dos cursos da área da saúde da Universidade do Estado do Amazonas quanto ao uso das tecnologias da comunicação e informação, bem como os fatores que influenciam na escolha de recursos para os seus respectivos estudos. Nesse contexto são abordados dados sociodemográficos, tipos de recursos utilizados para o estudo, conhecimento sobre a UNA-SUS, dentre outras variáreis. Adicionalmente, é discutida a necessidade de estímulo ao uso dos recursos educacionais que a UNA-SUS oferece, que são potencialmente eficazes à nível nacional quanto à formação e capacitação dos profissionais da saúde visando o fortalecimento do ensino na modalidade a distância.

Palavras-chave: perfil do usuário, recursos educacionais, educação em saúde

\section{AGRADECIMENTOS}

AGRADECEMOS AOS PARTICIPANTES DA PESQUISA, À UNIVERSIDADE DO ESTADO DO AMAZONAS (UEA) E AO PATROCINADOR DO PROJETO UNA-SUS AMAZÔNIA. 


\section{INTRODUÇÃO}

O Sistema Único de Saúde (SUS) brasileiro tem por objetivo atender as necessidades de assistências em saúde e a formação de recursos humanos na área de saúde do país. O SUS está amparado pelos princípios da universalidade, integralidade e equidade, que tem por objetivo tornar a assistência em saúde um direito amplo, necessitando, portanto, de constantes investidas do governo no sentido de estender e aprimorar o atendimento da população (MINISTÉRIO DA SAÚDE DO BRASIL, 2018).

"Para atender às necessidades de capacitação e educação permanente dos profissionais de saúde que atuam no SUS, o Ministério da Saúde criou em 2010 a Universidade Aberta do SUS (UNA-SUS). A UNA-SUS é composta por três pilares estruturais: 35 universidades, que trabalham, de forma colaborativa, planejando e desenvolvendo cursos na modalidade $\mathrm{EaD}$, recursos educacionais e tecnologias que apoiam a realização e manutenção de tais cursos; uma coleção de recursos educacionais em saúde" (ARES1), onde todo o conteúdo educacional produzidos pela UNA-SUS está disponível; e a "plataforma AROUCA, um sistema web que concentra dados educacionais e profissionais dos especialistas em saúde do SUS e ainda, oferece diversificados cursos, de caráter prático e acadêmico" (SANTOS et al., 2017).

Com a necessidade de identificar o perfil educacional dos profissionais e estudantes de saúde visando o desenvolvimento e aprimoramento de ferramentas de ensino presentes nas plataformas virtuais da rede UNA-SUS, fez-se necessário analisar o comportamento dos profissionais de saúde quanto ao uso e exploração de tecnologias pedagógicas digitais ao longo de sua formação continuada. Este trabalho aborda sobre a condução e análise de um estudo exploratório realizado em 2017 com estudantes e profissionais da área de saúde da Escola Superior de Ciências da Saúde da Universidade do Estado do Amazonas (UEA).

Como contribuições deste trabalho são destacados: 1 . Estudo de campo acerca do perfil educacional dos profissionais da área de saúde; 2 . Identificação de pontos positivos e negativos nas interações digitais dos usuários; 3. Discussão acerca do uso de tecnologias digitais no ensino da área de saúde.

\section{TRABALHOS RELACIONADOS}

O processo migratório da economia industrial da informação para a economia da informação em rede, tem implicado na mudança de um modelo altamente dependente do capital físico, para um novo padrão no qual os indivíduos conectam-se por meio da 
rede mundial de computadores, o que possibilita a criação e a difusão de conhecimento de forma extremamente dinâmica. Em conformidade com esta tendência, na área da saúde, já é possível enumerar diversos portais de ensino e disseminação do conhecimento, com acesso gratuito, como o VEDUCA e o SUPERCOURSE (ROCHA, 2015).

"Em uma vertente paralela à dos portais considerados institucionalizados ou formais, verifica-se um fervilhante movimento de difusão de conhecimento em saúde a partir dos portais considerados informais, como a rede social FACEBOOK e o portal YouTube". Uma pesquisa realizada entre docentes e discentes de uma universidade brasileira, "revelou que um número expressivo dos indivíduos entrevistados utilizava o FACEBOOK para atividades relacionadas às disciplinas dos cursos de graduação, como por exemplo, a troca de materiais, organização da rotina de estudos e postagens de conteúdos" (ALMEIDA FILHO, 2013; POSSOLLI; DO NASCIMENTO; DA SILVA, 2015).

Almeida Filho (2013), destaca a importância dos disseminadores informais de conhecimento, tal qual o YouTube e a Wikipédia. Afirma que a "insegurança" das informações contidas nestas plataformas, acabam por conduzir a um processo contínuo de contestação e validação das informações ali disponibilizadas, ao contrário dos modelos nos quais os conteúdos são chancelados e cristalizados por determinada autoridade intelectual, modelos estes que, segundo o autor já se encontram ultrapassados.

Os avanços tecnológicos, acorridos nos últimos anos, permite grandes oportunidades de acesso às informações. Tal contexto propicia a criação de ferramentas tecnológicas capazes de promover a integração e aperfeiçoamento educacional de diversos profissionais, inclusive àqueles que atuam na área de saúde. Neste cenário, Silva et al. (2015) afirmam que a incorporação do ensino a distância contribuiria para potencializar os programas de educação permanente e possibilitar, ao mesmo tempo, o desenvolvimento pessoal daqueles que trabalham na área da saúde. No mesmo trabalho, Silva conclui que o uso da $\mathrm{EaD}$ tem tido uma importante contribuição para o desenvolvimento dos recursos humanos em saúde.

Ribas et al. (2017), apresenta um estudo com objetivo de "analisar a visão da sociedade com relação a aceitação do profissional da área da saúde com formação de cursos EaD". Ele conclui que a maioria dos entrevistados não mostraram preconceito com profissionais formados nessa modalidade, e que afirmam ainda já terem buscado informações sobre cursos EaD. 


\section{METODOLOGIA}

O delineamento deste estudo se caracteriza pelo caráter descritivo. Nessa perspectiva, Zanella (2009) afirma que a principal preocupação deste método se refere à descrição de fenômenos por meio dos significados transmitidos pelo ambiente. Ou seja, não existe a preocupação de explicar os fenômenos descritos, ainda que sirva de suporte para a interpretação.

Pretendendo simplificar o tratamento dos dados, utilizou-se o método de regressão logística (PAGANO; GAUVREAU, 2010). Inicialmente foram testadas as associações binárias entre a variável dependente e cada uma das variáveis independentes. Este procedimento foi repetido individualmente para cada um dos recursos educacionais constantes no instrumento de coleta de dados (Quadro 1). Em seguida, procedia-se análise de regressão logística com múltiplas variáveis, incluindo-se, além da variável dependente (recurso educacional), todas aquelas que apresentaram valor de $p \leq 0,20$, na análise binária. Os modelos de regressão múltipla, referentes a cada um dos recursos educacionais, foram ajustados por meio da técnica stepwise backward, até a obtenção do melhor modelo ajustado (SANTOS et al, 2015). Ao final da análise, foram consideradas significativos, os resultados cujo valor de $p \leq 0,05$ (PAGANO; GAUVREAU, 2010).

Quadro 1 - Variáveis em estudo

\begin{tabular}{|c|c|}
\hline Variável & Categoria \\
\hline Gênero & $\begin{array}{l}1 \text { - Feminino } \\
2 \text { - Masculino } \\
3 \text { - Outro }\end{array}$ \\
\hline Ocupação & $\begin{array}{l}1 \text { - Profissional } \\
2 \text { - Estudante }\end{array}$ \\
\hline Área de atuação & $\begin{array}{l}\text { 1- Farmácia } \\
2 \text { - Educação Física } \\
3 \text { - Enfermagem } \\
4 \text { - Medicina } \\
5 \text { - Odontologia } \\
6 \text { - Psicologia }\end{array}$ \\
\hline \multicolumn{2}{|c|}{ Recursos utilizados para estudo } \\
\hline Livro & $\begin{array}{l}0 \text { - Não } \\
1 \text { - Sim }\end{array}$ \\
\hline Artigos Científicos & $\begin{array}{l}0 \text { - Não } \\
1 \text { - Sim }\end{array}$ \\
\hline E-books & $\begin{array}{l}0 \text { - Não } \\
1 \text { - Sim }\end{array}$ \\
\hline Videos & $\begin{array}{l}0-N a ̈ o \\
1-\operatorname{Sim}\end{array}$ \\
\hline Documentos governamentais (cartilhas, folhetos) & $\begin{array}{l}0 \text { - Não } \\
1 \text { - Sim }\end{array}$ \\
\hline $\begin{array}{l}\text { Fichamentos referentes a conteúdos não documentais } \\
\text { (eventos, palestras, workshops) }\end{array}$ & $\begin{array}{l}0-N a ̈ o \\
1-S i m\end{array}$ \\
\hline Imagens & $\begin{array}{l}0 \text { - Não } \\
1 \text { - Sim }\end{array}$ \\
\hline \multicolumn{2}{|c|}{ Quesitos adotados para confiar em um site ou conteúdo online } \\
\hline $\begin{array}{l}\text { Terminologia/nomenclatura utilizada no site, conteúdo ou } \\
\text { recurso }\end{array}$ & $\begin{array}{l}0-N a ̃ o \\
1-\operatorname{Sim}\end{array}$ \\
\hline Fontes bibliográficas & $\begin{array}{l}0 \text { - Não } \\
1 \text {-Sim }\end{array}$ \\
\hline Instituição ou empresa mantenedora & $\begin{array}{l}0 \text { - Nắ } \\
1 \text { - Sim }\end{array}$ \\
\hline Tipo de recurso disponivel para consulta & $\begin{array}{l}0-\text { Não } \\
1-\text { Sim }\end{array}$ \\
\hline Indicação de amigos & $\begin{array}{l}0 \text { - Não } \\
1 \text {-Sim }\end{array}$ \\
\hline Indicação de professores & $\begin{array}{l}\text { O-Năo } \\
1-\operatorname{sim}\end{array}$ \\
\hline Formato do arquivo & $\begin{array}{l}0 \text { - Não } \\
1 \text { - Sim }\end{array}$ \\
\hline
\end{tabular}

Fonte: Autores, 2017 
Quanto ao recorte temporal, o método utilizado foi o transversal - ou seja, realizado em um único momento devido à não necessidade de acompanhamento ao longo de um período de tempo. A pesquisa foi realizada durante o primeiro semestre de 2017 , sendo realizada inicialmente em abril e posteriormente em maio.

A amostra foi composta por 100 indivíduos entre profissionais e estudantes da área da saúde. A seleção dos mesmos se deu por critério de conveniência, uma vez que foram convidados, aqueles que transitavam pela Universidade no momento da coleta de dados pelos pesquisadores. Para a coleta de dados, utilizou-se formulário eletrônico, disponibilizado em dispositivo móvel do formato tablet, o qual foi preenchido pelo próprio participante de forma anônima.

O presente estudo foi realizado em conformidade com a resolução CNS 466/2012, obteve aprovação após análise pelo Comitê de Ética em Pesquisa (CEP) da Escola Superior de Ciências da Saúde - ESA/UEA via Plataforma Brasil, sob CAAE nº 71903617.9.0000.5016 com aprovação sob o parecer nํㅜㄴ.352.456.

\section{RESULTADOS OBTIDOS}

A seguir são mostrados os resultados obtidos através da investigação feita com objetivo de identificar o comportamento dos profissionais e estudantes da saúde quanto a utilização e aceitação de recursos/materiais virtuais no aprimoramento de seus estudos.

Quanto ao perfil dos respondentes, foi verificado que a maioria dos entrevistados correspondem ao sexo feminino $-56 \%$ do total, conforme exposto na Tabela 1.Em relação à ocupação cerca de $82,83 \%$ são estudantes, sendo a Medicina, a grande área de atuação dos participantes - $65 \%$ do total. Verificou-se também sobre o conhecimento do participante quanto à Universidade Aberta do SUS - UNA-SUS e constatou-se que $37 \%$ dos entrevistados não conhecem de fato o que é a UNA-SUS. Além disso, foi perguntado se conheciam sobre o formato de recurso educacional em SCORM e o PPU (Pacote Padrão UNA-SUS) e mais de $90 \%$ dos entrevistados não possuem conhecimento destes. Considera-se este índice elevado, uma vez que a UNA-SUS é uma rede nacional gratuita e específica para os profissionais da área de saúde, sejam estudantes ou profissionais de fato, a qual possui plataformas com recursos para estudo, além de capacitações permanentes.

Tabela 1- - Identificação dos participantes por categoria de pesquisa. 


\begin{tabular}{lcc}
\hline Variável & $\mathbf{n}$ & $\%$ \\
\hline Gênero & 56 & 56,00 \\
$\quad$ Feminino & 42 & 42,00 \\
$\quad$ Masculino & 2 & 2,00 \\
$\quad$ Outro & 17 & 17,17 \\
Ocupação & 82 & 82,83 \\
$\quad$ Profissional & & \\
$\quad$ Estudante & 1 & 1,00 \\
Área de atuação & 14 & 14,00 \\
$\quad$ Educação física & 65 & 65,00 \\
$\quad$ Enfermagem & 17 & 17,00 \\
Medicina & 3 & 3,00 \\
$\quad$ Odontologia & & \\
$\quad$ Psicologia & 37 & 37,00 \\
Conhece a UNA-Sus & 36 & 36,00 \\
$\quad$ Não & 27 & 27,00 \\
$\quad$ Sim & & \\
$\quad$ Já ouviu falar &
\end{tabular}

Fonte: Autores, 2017.

Em relação aos recursos educacionais que os entrevistados utilizam para seus estudos, observa-se no Gráfico 1 que mais de 95\% dos entrevistados utilizam "livros" como material de estudos, além de "artigos científicos" - cerca de $75 \%$, isso quer dizer que estes indivíduos preferem estudar por meio de textos mais estruturados com poucas ilustrações e interações como os vídeos, os quais hoje em dia são bastante utilizados na educação, além da aplicabilidade da gamificação no processo de ensino-aprendizagem que está quebrando os paradigmas da educação tecnológica.

Gráfico 1- Porcentagem das categorias de materiais de estudo

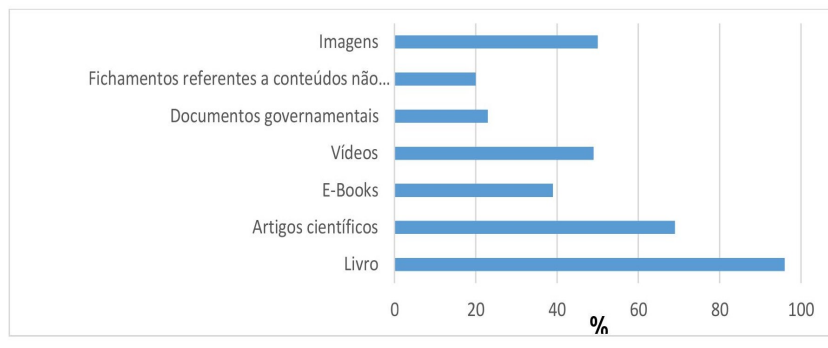

Fonte: Autores, 2017.

Quando questionados sobre a confiabilidade de um conteúdo online ou site, a maioria dos participantes da pesquisa consideram que as "fontes bibliográficas" e a "indicação de professores" são as categorias que mais influenciam na escolha do recurso a ser utilizado, aproximadamente $83 \%$ e $73 \%$, respectivamente, conforme demonstra 0 Gráfico 2.

Gráfico 2 - Porcentagem das categorias que influenciam na confiabilidade de um recurso educacional. 


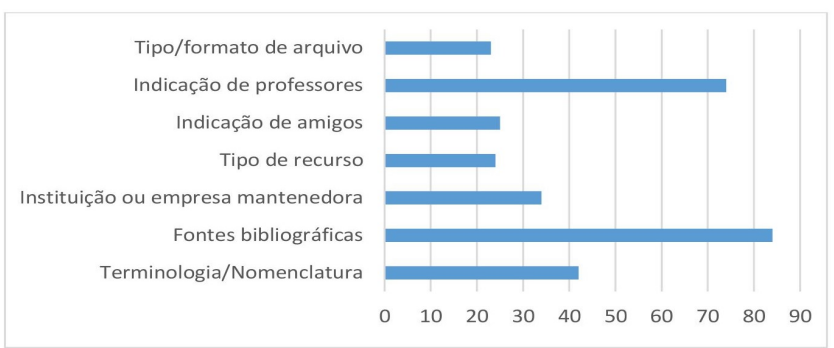

Fonte: Autores, 2017.

Os odds ratios resultantes dos três modelos de regressão logística com o emprego de múltiplas variáveis é apresentado na Tabela 2.

Tabela 2 - Odds ratios (OR) entre recurso utilizado para estudo e variáveis sociodemográficas e quesitos adotados para confiar em conteúdos online ou sites.

\begin{tabular}{|c|c|c|c|c|c|}
\hline Variável & $\mathbf{n}$ & OR & IC $95 \%$ & & p \\
\hline \multicolumn{6}{|c|}{ RECURSO UTILIZADO: Artigo Científico } \\
\hline \multicolumn{6}{|c|}{$\begin{array}{l}\text { Quesito para confiar em recurso online: Fonte } \\
\text { Bibliográfica }\end{array}$} \\
\hline Não & 5 & & & & \\
\hline Sim & 64 & 7,04 & 2,18 & 22,69 & 0,00 \\
\hline \multicolumn{6}{|c|}{$\begin{array}{l}\text { Quesito para confiar em recurso online: Tipo de } \\
\text { recurso }\end{array}$} \\
\hline Não & 48 & & & & \\
\hline sim & 21 & 4,08 & 1,12 & 14,93 & 0,03 \\
\hline \multicolumn{6}{|l|}{ RECURSO UTILIZADO: Vídeo } \\
\hline \multicolumn{6}{|l|}{ Gênero } \\
\hline Feminino & 23 & & & & \\
\hline Masculino & 26 & 2,52 & 1,03 & 6,15 & 0,04 \\
\hline Outro & 0 & -- & --- & -- & --- \\
\hline \multicolumn{6}{|c|}{$\begin{array}{l}\text { Quesito para confiar em recurso online: Fonte } \\
\text { Bibliográfica }\end{array}$} \\
\hline Não & 4 & & & & \\
\hline Sim & 45 & 4,50 & 1,23 & 16,41 & 0,02 \\
\hline \multicolumn{6}{|c|}{$\begin{array}{l}\text { Quesito para confiar em recurso online: Tipo de } \\
\text { recurso }\end{array}$} \\
\hline Não & 32 & & & & \\
\hline Sim & 17 & 2,73 & 0,96 & 7,77 & 0,60 \\
\hline \multicolumn{6}{|l|}{ RECURSO UTILIZADO: Imagem } \\
\hline \multicolumn{6}{|c|}{$\begin{array}{l}\text { Quesito para confiar em recurso online: } \\
\text { Nomenclatura }\end{array}$} \\
\hline Não & 24 & & & & \\
\hline Sim & 26 & 2,98 & 1,18 & 7,57 & 0,02 \\
\hline \multicolumn{6}{|c|}{$\begin{array}{l}\text { Quesito para confiar em recurso online: } \\
\text { Instituição mantenedora }\end{array}$} \\
\hline Não & 27 & & & & \\
\hline Sim & 23 & 3,67 & 1,38 & 9,76 & 0,01 \\
\hline \multicolumn{6}{|c|}{$\begin{array}{l}\text { Quesito para confiar em recurso online: } \\
\text { Indicação de amigos }\end{array}$} \\
\hline Não & 30 & & & & \\
\hline Sim & 20 & 6,83 & 2,14 & 21,85 & 0,00 \\
\hline
\end{tabular}

Fonte: Autores, 2017.

O modelo 1, investigou a associação entre a variável dependente "uso de artigo científico como material de estudo" e as variáveis independentes (gênero, ocupação, área de atuação e os quesitos adotados para confiar em um conteúdo online) e, 
conforme se verifica, o modelo final ajustado, aponta que o uso de artigos científicos está relacionado com a adoção dos quesitos "fonte bibliográfica" e "tipo de recurso". Considera-se que o sujeito que faz uso de artigos científicos tende a atentar-se às fontes bibliográficas como quesito para confiar no conteúdo online, cerca de sete vezes mais do que o indivíduo que não faz uso de artigos científicos como material de estudo.

Para o modelo 2 de regressão logística, observou-se que os homens possuem 2,5 vezes mais chances do que as mulheres de utilizarem os recursos de vídeo, como material de aprendizado. Além disso, consideram como quesito de confiabilidade em conteúdo online, a "instituição mantenedora do canal" e a "indicação de amigos". Já em relação aos que relataram fazer o uso de imagem como recurso de estudo, verificou-se que os quesitos de confiabilidade do conteúdo são: a terminologia ou nomenclatura utilizada, a instituição mantenedora e a indicação de amigos.

Por fim, buscou-se apurar a relação entre o conhecimento prévio da UNA-SUS com as demais variáveis (gênero, ocupação, área de atuação, recursos utilizados para estudo e quesitos adotados para confiar em conteúdo online). E, como resultado observou-se que estão associados ao conhecimento anterior da UNA- SUS, o fato de ser profissional formado; a não utilização de fichamentos referentes a conteúdos não documentais como material de estudo; a adoção de fontes bibliográficas e a indicação não proveniente de amigos como quesitos de confiabilidade em conteúdo online.

\section{CONCLUSÕES}

A partir desta pesquisa, foi possível analisar questões voltadas a compreensão de usabilidade de recursos educacionais tecnológicos na concepção de docentes e discentes da Escola Superior de Ciências da Saúde-ESA/UEA.

Nesse aspecto, o resultado geral dos critérios aponta que o perfil dos usuários ainda é "cartesiano", ou seja, utilizam fontes de recursos educacionais considerados "arcaicos", como livros e artigos científicos, os quais não possuem nenhum tipo de interação com o usuário, e que atualmente não está convergindo com o avanço tecnológico. Tal característica pode estar relacionada com a falta de conhecimento do próprio docente que não utiliza a tecnologia em sala de aula e consequentemente não instiga o aluno à utilizar ferramentas qualificadas e tecnológicas nos estudos.

Destaca-se a importância de pensar em soluções que possibilitem aos aplicativos UNASUS uma experiência cada vez mais personalizada com seus usuários. Sugere-se a exploração de dados com informações acerca dos próprios usuários para o 
desenvolvimento de sistemas capazes de capturar o objetivo destes e direcioná-los mais facilmente aos itens de seu interesse. Foi validada a hipótese da existência de um alto grau de interesse do público abordado em aplicativos e novas tecnologias educacionais.

Desta forma, os resultados demonstraram que ainda há muito trabalho de divulgação dos recursos ofertados pela UNA-SUS. Ressaltamos que é imprescindível que haja futuras pesquisas que continuem a averiguar, contribuir e avaliar a eficiência do acervo, dos serviços ofertados e da satisfação dos usuários. Os resultados desta pesquisa fomentarão o desenvolvimento e aprimoramento de ferramentas de ensino presentes nas plataformas virtuais da rede UNA-SUS, já que, dentro da rede, existem universidades que produzem cursos educacionais e ainda universidades parceiras que desenvolvem tecnologias voltadas a apoiar e atender o objetivo maior da rede: capacitação continuada de profissionais da saúde.

\section{REFERÊNCIAS}

ALMEIDA FILHO, N. M. DE. Contextos, impasses e desafios na formação de trabalhadores em Saúde Coletiva no Brasil. Ciência \& Saúde Coletiva, v. 18, n. 6, p. 1677-1682, 2013.

MINISTÉRIO DA SAÚDE DO BRASIL. Sistema Único de Saúde. Disponível em: . Acesso em: 23 jan. 2018.

PAGANO, M.; GAUVREAU, K. Princípios de bioestatística. Tradução Luiz Sérgio de Castro Paiva. Revisão técnica Lúcia Pereira Barroso. São Paulo: Cengage Learning, 2010.

POSSOLLI, G. E.; DO NASCIMENTO, G. L.; DA SILVA, J. O. M. A Utilização do Facebook no Contexto Acadêmico: o Perfil de Utilização e as Contribuições Pedagógicas e para Educação em Saúde. Renote, v. 13, n. 1, p. 1-10, 2015.

RIBAS, J. L. C. et al. A visão da sociedade para os profissionais com formação ead: a quebra de um paradigma. Revista on line de Política e Gestão Educacional, v. 21, n. esp3, p. 1598-1610, 2017.

ROCHA, J. S. Y. Use of information and communication technologies in health education. Problems and development. Medicina (Brazil), v. 48, n. 3, p. 214-223, 2015.

SANTOS, W. O. M. DOS et al. Telehealth and tele-education using mobile platforms- 
UNA-SUS Amazônia. Journal of the International Society for Telemedicine and EHealth, v. 5, p. 3-6, 2017.

SILVA, A. DAS N. et al. Limites e possibilidades do ensino à distância (EaD) na educação permanente em saúde: revisão integrativa. Ciência \& Saúde Coletiva, v. 20, n. 4, p. 1099-1107, 2015.

ZANELLA, L. C. H. Metodologia de Estudo e de Pesquisa em Administração. [s.l: S.n.]. 